\title{
The Surrogate Method: Past, Present and Future
}

S. R. Lesher, L. A. Bernstein, J. T. Burke, B. F. Lyles, R. M. Clark, P. Fallon, L. Phair

January 29, 2008

4th International Conference on Fission and Properties of Neutron-Rich Nuclei Sanibel, FL, United States November 10, 2007 through November 17, 2007 
This document was prepared as an account of work sponsored by an agency of the United States government. Neither the United States government nor Lawrence Livermore National Security, LLC, nor any of their employees makes any warranty, expressed or implied, or assumes any legal liability or responsibility for the accuracy, completeness, or usefulness of any information, apparatus, product, or process disclosed, or represents that its use would not infringe privately owned rights. Reference herein to any specific commercial product, process, or service by trade name, trademark, manufacturer, or otherwise does not necessarily constitute or imply its endorsement, recommendation, or favoring by the United States government or Lawrence Livermore National Security, LLC. The views and opinions of authors expressed herein do not necessarily state or reflect those of the United States government or Lawrence Livermore National Security, LLC, and shall not be used for advertising or product endorsement purposes. 


\title{
The Surrogate Method; Past, Present and Future*
}

\author{
S.R. Lesher*, L.A. Bernstein, J.T. Burke, B.F. Lyles \\ Lawrence Livermore National Laboratory, Livermore, California 94551 \\ *E-mail: lesher1@llnl.gov \\ R.M. Clark, P. Fallon, L. Phair \\ Lawrence Berkeley National Laboratory, Berkeley, California 94720
}

The STARS/LiBerACE collaboration has been exploring the surrogate technique with success in the actinide region. This method uses a direct reaction to measure the decay probability of the same compound nucleus produced via a neutron-induced channel. This paper serves as an overview of these activities. Using the STARS array at 88-inch Cyclotron at Lawrence Berkeley National Laboratory we have explored the following surrogate reactions: ${ }^{234} \mathrm{U}\left(\alpha, \alpha^{\prime} f\right)$, ${ }^{235} \mathrm{U}\left({ }^{3} \mathrm{He}, \alpha f\right),{ }^{236} \mathrm{U}\left(\alpha, \alpha^{\prime} f\right),{ }^{238} \mathrm{U}\left(\alpha, \alpha^{\prime} f\right),{ }^{238} \mathrm{U}\left({ }^{3} \mathrm{He}, \alpha f\right),{ }^{238} \mathrm{U}\left({ }^{3} \mathrm{He}, t f\right)$ surrogates for ${ }^{233} \mathrm{U}(n, f),{ }^{233} \mathrm{U}(n, f),{ }^{235} \mathrm{U}(n, f),{ }^{237} \mathrm{U}(n, f),{ }^{236} \mathrm{U}(n, f)$, and ${ }^{237} \mathrm{~Np}(n, f)$, respectively.

Keywords: surrogate reactions, fission probability

\section{Introduction}

The Absolute Surrogate Technique was first suggested by Cramer and Britt in $1970^{1}$ to overcome the problem associated with neutron induced experiments on short-lived targets. The main assertion of the Surrogate Technique is that the fission probability is independent of the direct reaction used to populate the compound nucleus of interest. If similar states are excited in the neutron capture and direct reactions, then the cross section for neutron fission can be estimated:

$$
\sigma_{(n, f)}\left(E_{n}\right) \approx P_{f}\left(E_{n}-S_{n}\right) \times \sigma_{C N}\left(E_{n}\right)
$$

where $\mathrm{E}_{n}$ is the equivalent neutron energy, $\mathrm{S}_{n}$ is the neutron separation energy and $\sigma_{C N}$ is the energy dependent compound nucleus formation cross

*This work was performed under the auspices of the U.S. Department of Energy by Lawrence Livermore National Laboratory in part under Contract W-7405-Eng-48 and in part under Contract DE-AC52-07NA27344. 
section. This equation depends on two factors (1) the $\sigma_{C N}$ is known from a model or another source and (2) the decay probability is independent of the spin and parity.

In the following years, they had limited success with this method in the actinide region. This absolute surrogate method was limited to an excitation energy of around 11-12 MeV because of the maximum beam energy available and the introduction of the carbon and oxygen contaminants at high energies.

The external surrogate ratio method (ESRM) was introduced 35 years later by Plettner et al..$^{2}$ to overcome some problems of the absolute method. With the ESRM, the same direct reaction is used to produce two different compound nuclei, the exit channel probabilities are measured, and the ratio is used to infer one cross section if the other is well-known. In this paper, the probability of the ${ }^{238} \mathrm{U}\left(d, d^{\prime} f\right) /{ }^{236} \mathrm{U}\left(d, d^{\prime} f\right)$ ratio was used as a surrogate for the ratio of the ${ }^{237} \mathrm{U}(n, f) /{ }^{235} \mathrm{U}(n, f)$ cross section and ${ }^{236} \mathrm{U}(d, p f) /{ }^{238} \mathrm{U}(d, p f)$ ratio was used as a surrogate for the ${ }^{236} \mathrm{U}(n, f) /{ }^{238} \mathrm{U}(n, f)$. However, these experiments still suffered from a 15 $-20 \%$ uncertainty at low energies.

The STARS-LiBerACE collaboration is now exploring the techniques more thoroughly with experimental and theoretical work. This paper serves as an overview of the experimental work performed by the group in the actinide region. The theoretical work has been explained in great detail by Escher and Dietrich. ${ }^{3}$

\section{Experimental}

In the experiments summarized here, the targets were bombarded with the desired particle beam produced by the 88 -inch Cyclotron at Lawrence Berkeley National Laboratory. The Silicon Telescope Array for Reaction Studies (STARS) was used to detect the scattered particles in coincidence with the outgoing fission fragments. The STARS array consisted of three to five Silicon Micron S2 detectors including a downstream $\Delta \mathrm{E}$ - E particle telescope where the $\Delta \mathrm{E}$ detector has a thickness of either $140 \mu \mathrm{m}$ or $500 \mu \mathrm{m}$ and the E detectors are in multiples of $1000 \mu \mathrm{m}$ and an upstream "fission" detector $(140 \mu \mathrm{m})$ as shown in Fig. 1. The S2 detectors can be set up with the full 48 rings and 16 sectors configuration or with a total of 24 rings and 8 sectors depending on the experiment and cover an angular range of $\theta_{\text {polar }}=10^{\circ}-66^{\circ}$ at one time with respect to the beam axis depending on the distance the telescope is placed from the target. Fission fragments were detected over an angular range of $\theta_{\text {polar }}=106^{\circ}-131^{\circ}$ upstream of 


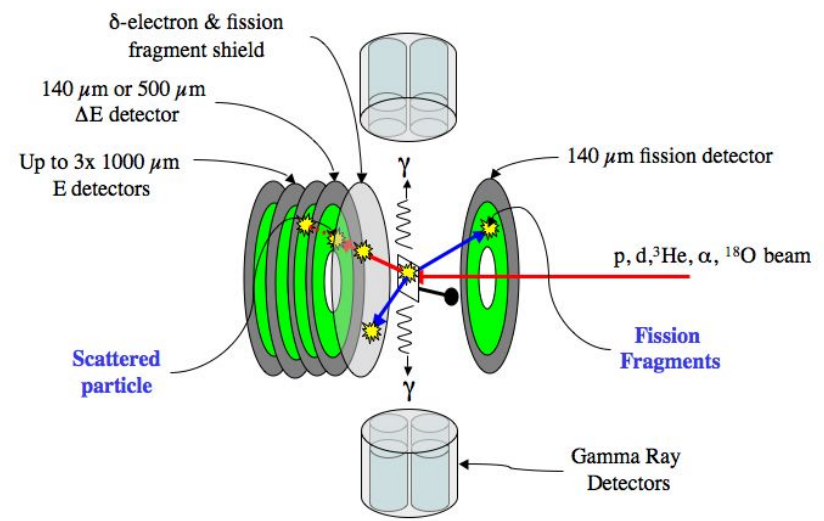

Fig. 1. (color on-line) A drawing of the STARS telescope array shown with the GeHP detectors LiBerACe.

the target. One of the master triggers (MT) set for the data acquisition can require a coincidence between the $\Delta \mathrm{E}$ and $\mathrm{E}$ detectors. Fission detector energies were recorded if they came typically within $4 \mu$ s of the trigger. In addition, the relative time difference between the $\Delta \mathrm{E}-\mathrm{E}$ detectors and the fission detector were recorded reducing the prompt window down to $\sim 200$ $-300 \mathrm{~ns}$. The setup is explained in more detail in Ref. ${ }^{4,5}$

In subsequent off line analysis, charged particles $\left(p, d, t,{ }^{3} \mathrm{He},{ }^{4} \mathrm{He},{ }^{16} \mathrm{O}\right)$ were identified by plotting the energy loss measured in the $\Delta \mathrm{E}$ detector against the total energy, $\Delta \mathrm{E}+\mathrm{E}$. A 2-d gate was employed to select the particle events of interest. The scattered particle energy was used, on an event by event basis, to determine the excitation energy of the uranium nucleus by correcting for the angular-dependent recoil energy of the target nucleus, for the energy losses in the target layers, and the thin $\left(4 \mathrm{mg} / \mathrm{cm}^{2}\right)$ aluminum fission fragment shield and the dead layers of the silicon detectors.

\section{Results}

An overview of the experiments conducted by the Livermore group and collaborators include three completed analysis and three experiments in various stages of completion in the actinide region.

In one of the first experiments, ${ }^{236} \mathrm{U}\left(184 \mu \mathrm{g} / \mathrm{cm}^{2}\right)$ and ${ }^{238} \mathrm{U}(585$ $\left.\mu \mathrm{g} / \mathrm{cm}^{2}\right)$ targets were bombarded with a $55 \mathrm{MeV} \alpha$ beam. ${ }^{236} \mathrm{U}\left(\alpha, \alpha^{\prime} f\right)$ is 
the surrogate for ${ }^{235} \mathrm{U}(n, f)$ and ${ }^{238} \mathrm{U}\left(\alpha, \alpha^{\prime} f\right)$ is the surrogate for ${ }^{237} \mathrm{U}(n, f)$. The cross section for neutrons on ${ }^{235} \mathrm{U}$ is well known but neutrons on ${ }^{237} \mathrm{U}$ was difficult to measure given the short half-life of the target $\left(\tau_{1 / 2}=6.75\right.$ days). To overcome this difficulty, the External Surrogate Ratio was used to find the ${ }^{237} \mathrm{U}(n, f)$ cross section over an equivalent neutron energy range of $0-20 \mathrm{MeV}^{4}$

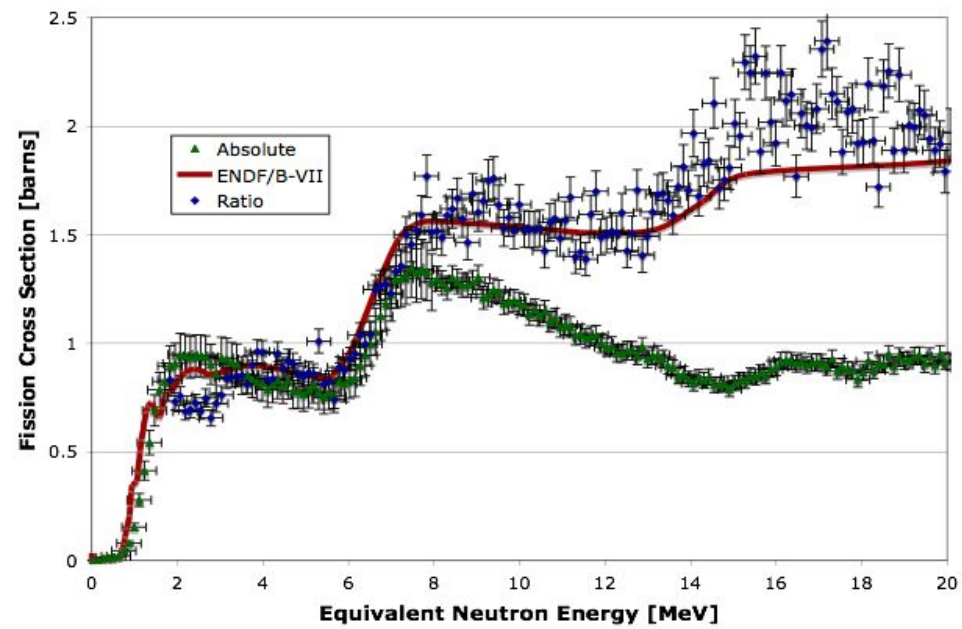

Fig. 2. (color on-line) The ${ }^{236} \mathrm{U}(n, f)$ cross section is plotted in three different ways. The solid line is from the $\mathrm{ENDF} / \mathrm{B}-\mathrm{VII}^{6}$ database, the green triangle points are from the absolute surrogate method while the blue diamonds are from the surrogate ratio method, both taken from Ref. ${ }^{5}$

In a second experiment, a $42 \mathrm{MeV}{ }^{3} \mathrm{He}$ beam was used on a 720 $\mu \mathrm{g} / \mathrm{cm}^{2}{ }^{235} \mathrm{U}$ target and a $761 \mu \mathrm{g} / \mathrm{cm}^{2}{ }^{238} \mathrm{U}$ target. The ${ }^{235} \mathrm{U}\left({ }^{3} \mathrm{He}, \alpha f\right)$ and ${ }^{238} \mathrm{U}\left({ }^{3} \mathrm{He}, \alpha f\right)$ were surrogates for ${ }^{233} \mathrm{U}(n, f)$ and ${ }^{236} \mathrm{U}(n, f)$, respectively. Using the alpha exit channel, the external ratio and the absolute surrogate techniques were used to examine the ${ }^{236} \mathrm{U}(n, f)$ cross section over an equivalent neutron energy range of 0 to $20 \mathrm{MeV}^{5}$ and the sensitivity to the spin and parity population distribution between the two entrance channels was explored. The results can be seen in Fig. 2. The data from the absolute surrogate technique agreed well with the direct measurement until the ${ }^{3} \mathrm{He}$ gate becomes mixed with the $\alpha$ gate and the denominator is affected. Significant deviations between the ESRM and the ENDF data are noticeable below about $3.3 \mathrm{MeV}$ and may indicate the breakdown of the WeisskophEwing assumption or a need to remeasure the ${ }^{233} \mathrm{U}(n, f)$ cross section in 
this region. Between the absolute surrogate technique, below $3.3 \mathrm{MeV}$ and the ESRM, above $3.3 \mathrm{MeV}$ we are able to reproduce the ENDF data curve.

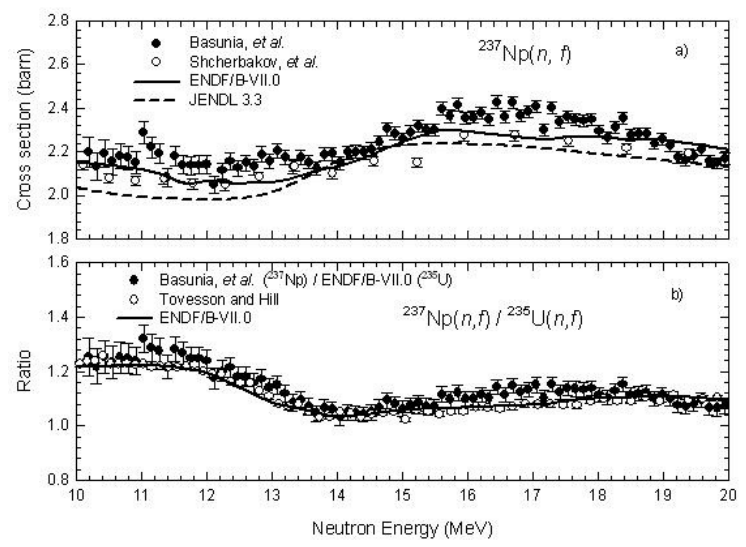

Fig. 3. (color on-line) a) The ${ }^{237} \mathrm{~Np}(n, f)$ cross section is plotted as an absolute surrogate and b) as a ratio to ${ }^{235} \mathrm{U}(n, f)$. Reproduced from Ref. ${ }^{7}$

From the same experiment, but using the ${ }^{238} \mathrm{U}\left({ }^{3} \mathrm{He}, t f\right)$ reaction channel, ${ }^{237} \mathrm{~Np}(n, f)$ was examined using the absolute surrogate technique for an energy range between $10-20 \mathrm{MeV}$ by Basunia et al. ${ }^{7} \mathrm{Fig}$. 3a) shows the results in comparison with the STARS data and the ENDF/B-VII.0 database and direct measurement data from Shcherbakov et al. ${ }^{8}$ These results agree within $6 \%$. In Fig. 3b), the STARS data is taken in ratio with the ${ }^{235} \mathrm{U}$ data from ENDF/B-VII.0 to compare with a recent direct measurement of the same cross section ${ }^{9}$ with good agreement. This work benchmarks the use of the $\left({ }^{3} \mathrm{He}, t f\right)$ reaction for the absolute surrogate $(n, f)$ cross section in the $10-20 \mathrm{MeV}$ range.

Currently there are three experiments under analysis in the actinide region. First, ${ }^{234} \mathrm{U}\left(\alpha, \alpha^{\prime} f\right)$ and ${ }^{236} \mathrm{U}\left(\alpha, \alpha^{\prime} f\right)$ serves as a benchmark for the ESRM since both of the surrogate reactions, ${ }^{233} \mathrm{U}(n, f)$ and ${ }^{235} \mathrm{U}(n, f)$, respectively have been measured directly. This will also confirm the result found in Burke et al. ${ }^{4,10}$

The next experiment involves bombarding a ${ }^{238} \mathrm{U}$ target with ${ }^{18} \mathrm{O}$ and observing the ${ }^{16} \mathrm{O}$ exit channel, to determine the ${ }^{239} \mathrm{U}(n, f)$ and $(n, 2 n)$ cross section as a test of the two-neutron transfer mechanism. ${ }^{11}$

Finally, a benchmark of the (n, $\gamma),(n, 2 n)$ using $21 \mathrm{MeV}$ and $29 \mathrm{MeV}$ deuterons on ${ }^{235} \mathrm{U}$ will test the Internal Surrogate Ratio Method (ISRM). In this technique, one compound nucleus is made and two exit channels are examined. As in the ESRM, if one of the exit channels is known, the 
other can be compared and cross section can be inferred. The ${ }^{235} \mathrm{U}(d, p x)$ reaction is the surrogate for ${ }^{235} \mathrm{U}(n, x)$, where $x=f, \gamma, 2 n$, etc. ${ }^{12}$

\section{Conclusions}

The STARS/LiBerACE collaboration has performed a variety of experiments to test the surrogate technique and benchmark the surrogate ratio method. The technique has been benchmarked for the $\left(\alpha, \alpha^{\prime}\right)$ and $\left({ }^{3} \mathrm{He}, t\right)$ reactions in different energy ranges. The ${ }^{237} \mathrm{U}(n, f)$ cross section was extracted from 0-20 MeV equivalent neutron energy with less than $10 \%$ uncertainty. We have explored the spin distribution probability between the direct and neutron induced reactions to test one of the major ansatz of the method. Other experiments are in various stages of analysis to determine new cross-sections in the actinide region and also benchmark different reactions.

\section{References}

1. J.D. Cramer and H.C. Britt, Nucl. Sci. Eng. 41, p. 177 (1970).

2. C. Plettner, H. Ai, C.W. Beausang, L.A. Bernstein, L. Ahle, H. Amro, M. Babilon, J.T. Burke, et al., Phys. Rev. C 71, p. 051602(R) (2005).

3. J. E. Escher and F. S. Dietrich, Phys. Rev. C 74, p. 054601 (2006).

4. J.T. Burke, L.A. Bernstein, J. Escher, L. Ahle, J.A. Church, F.S. Dietrich, K.J. Moody, E.B. Norman, L. Phair, et al., Phys. Rev. C 73, p. 054604 (2006).

5. B.F. Lyles, L.A. Bernstein, J.T. Burke, F.A. Dietrich, J. Escher, I. Thompson, D.L. Bleuel, R.M. Clark, P. Fallon, et al., Phys. Rev. C 76, p. 014606 (2007).

6. M.B. Chadwick, P. Obložinský, M. Herman, N.M. Greene, R.D. McKnight, D.L. Smith, P.G. Young, R.E. MacFarlane, et al., Nucl. Data Sheets 107, p. 2931 (2006).

7. M.A. Basunia, R.M. Clark, L. Phair, D.L. Bleuel, P. Fallon, J. Gibelin, M.A. McMahan, et al., Phys. Rev. C submitted (2007).

8. O. Shcherbakov, A. Donets, A. Evdokimov, A. Fomichev, T. Kukahori, A. Hasegawa, A. Laptev, V. Maslowv, G. Petrov, et al., Journal of Nucl. Sci. and Tech Supp. 2, p. 230 (2002).

9. F. Tovesson and T. Hill, Phys. Rev. C 75, p. 034610 (2007).

10. S.R. Lesher, L.A. Bernstein, H. Ai, C.W. Beausang, D.L. Bleuel, J.T. Burke, R.M. Clark, P. Fallon, J. Gibelin, I.Y. Lee, et al., analysis on going.

11. J.T. Burke, L.A. Bernstein, J. Escher, L. Ahle, F.S. Dietrich, E.B. Norman, L. Phair, P. Fallon, R.M. Clark, et al., analysis on going.

12. J.M. Allmond, S.R. Lesher, C.W. Beausang, L.A. Bernstein, D.L. Bleuel, J.T. Burke, L. Phair, P. Fallon, et al., analysis on going. 